Proceeding

\title{
Is an effective functional replacement of a lumbar segment possible? biokinemetrie as a surgical projection
}

Volume 8 Issue 4 - 2017

\author{
Rieger $B,{ }^{1,6}$ Jiang $H,{ }^{1,2}$ Polanski W, ${ }^{1}$ Molcanyi \\ $M,{ }^{3}$ Zivcak J, ${ }^{4}$ Reinshagen $C^{5}$ Brautferger $U,{ }^{6}$ \\ Mysliwiec P,' Schackert G,' Norbert Welsch ${ }^{6}$ \\ 'Department of Neurosurgery, University Hospital of Dresden, \\ Germany \\ 2Department of Neurosurgery, Chinese PLA General Hospital, \\ China \\ ${ }^{3}$ Institute of Neurophysiology, Medical Faculty, University of \\ Cologne, Germany \\ ${ }^{4}$ Department of Biomedical Engineering, Technical University of \\ Kosice, Slovakia \\ ${ }^{5}$ Department of Neurosurgery, Brigham and Women's Hospital, \\ Harvard Medical School, USA \\ ${ }^{6}$ Prospective Spine, USA
} column. During stabilizing operations, whether rigid or prosthetic, a fracture-like segmental release prior to instrumentation is currently the standard of care. However, as known from cases of natural fracture, unnatural movement patterns are possible which result in callus formation. Consequently, in order to create a biokinemetrically functional segmental replacement, physiological range of motion (ROM) must first be measured, followed by preoperative simulation of post-surgical conditions with adjustment to adjacent level ROM in order to prevent non-physiological segmental movement and undesired ossification.

\section{Methods}

After lumbar segmental movement characteristics of volunteers were described video-fluoroscopically, the movement of every lumbar segment was simulated. Software was used to automatically identify the worst segment, by analyzing patients spinal movements based on plain X-rays in inclination, neutral position, reclination, a.p. and oblique right/left. ROM analysis was repeated and the ROM of the best segment was projected into the worst segment in order to define the optimal height for segmental replacement regarding current degeneration (Figure 1). After software-projected optimization of segmental height, extrapolated characteristics were calculated to define placement and bearing surfaces of an interspinous device which supports the facet joints and reflects the natural segmental movement pattern (Figure 2-4).

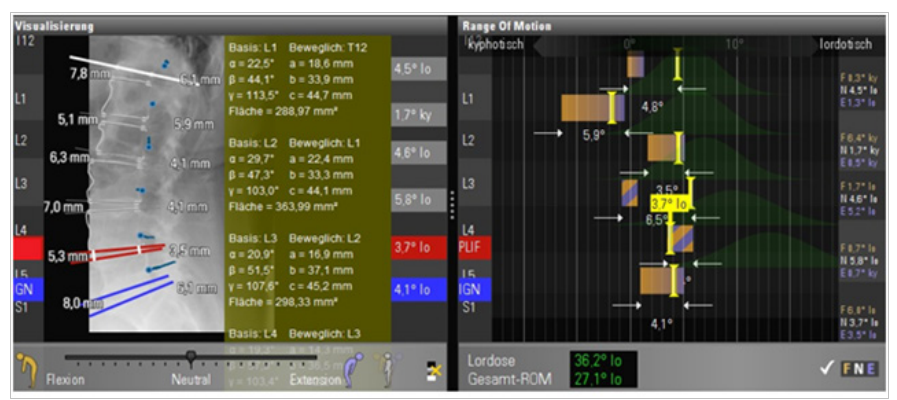

Figure I Segmental movement characteristics described with small blue curves in the plain X-ray.
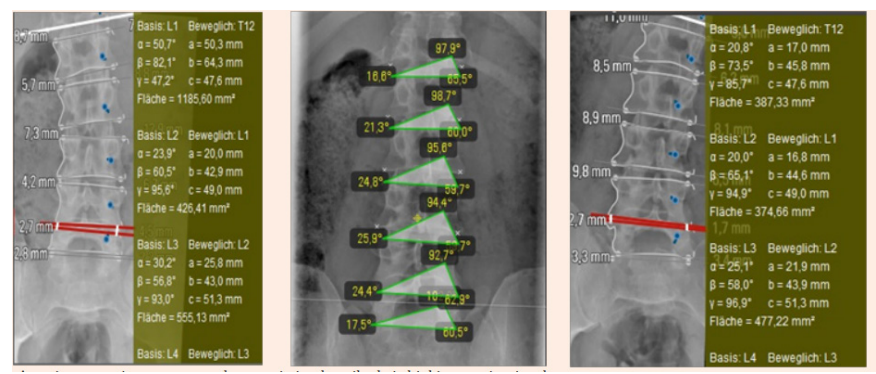

Figure 2 Anterior-posterior movement characteristics described via biokinemetric triangle.

A: X-ray in inclination with blue curves describing the probable movement of a point on the roof of the neuroforamen B: X-ray in neutral position $\mathrm{C}$ : X-ray in reclination green tables with data of the biokinemetric triangles, bar diagrams illustrate the common range of motion analysis.
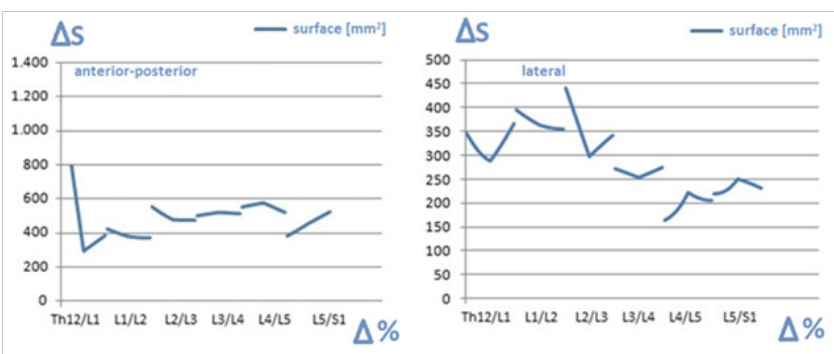


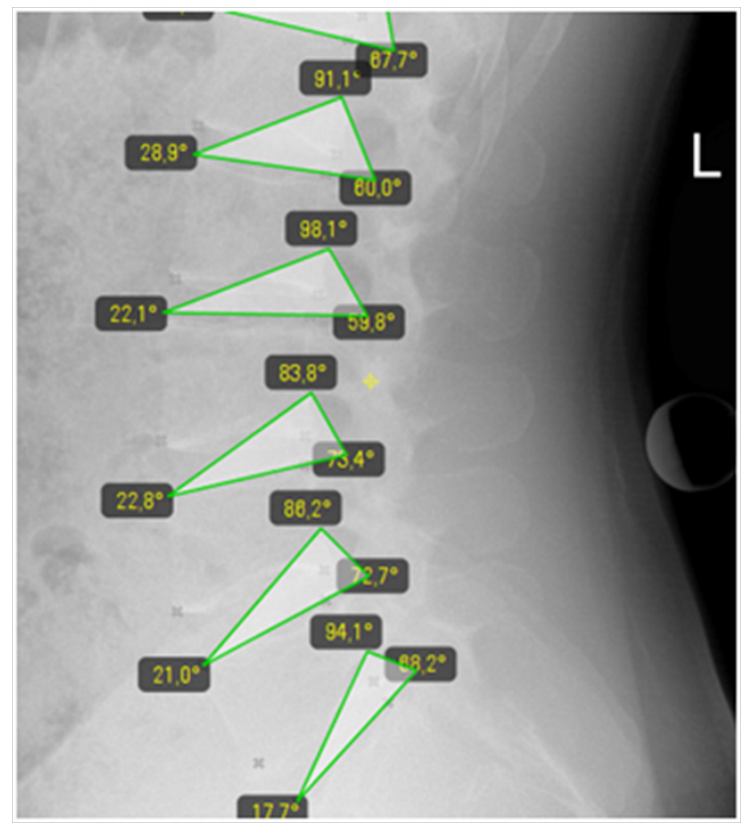

Figure 3 Lateral movement described via triangle.
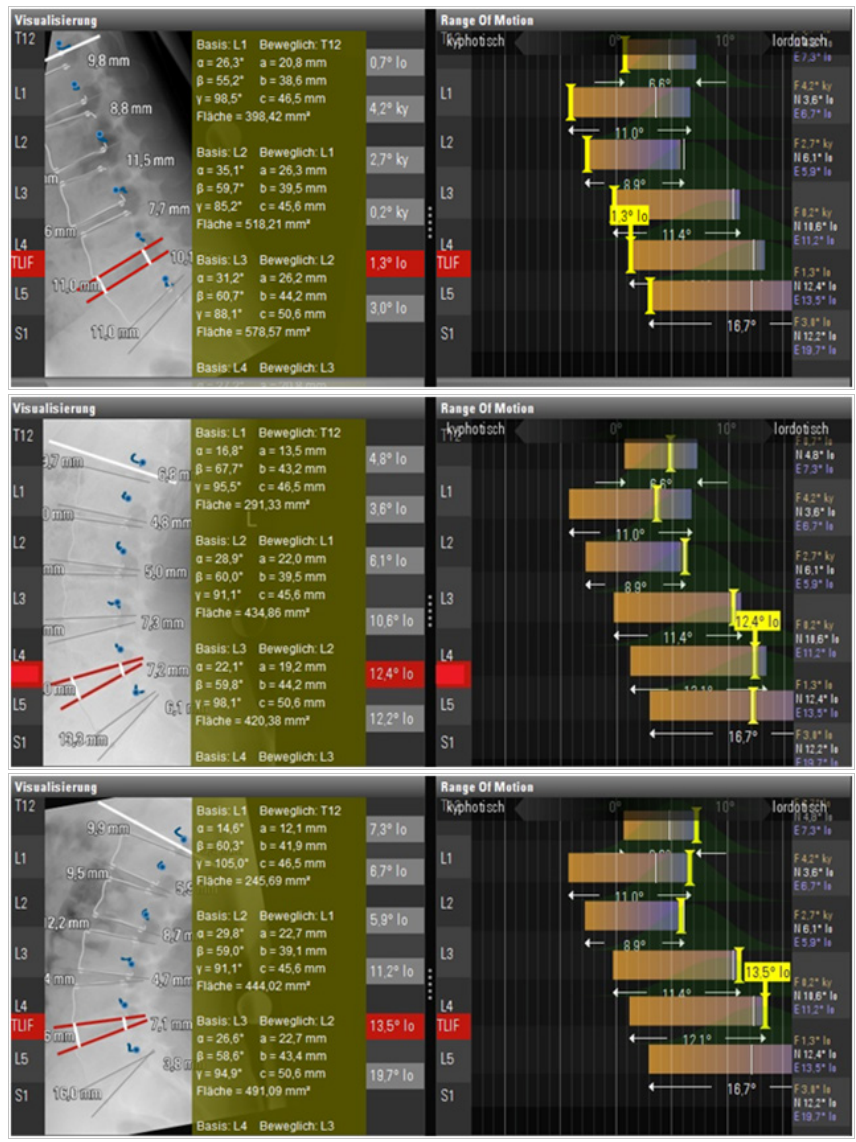

Figure 4 Lateral movement patterns of the lumbar spine in order to obtain information about the most probable movement of each segment using a surrogate model based on video-fluoroscopic examinations.

\section{Results}

The construction of an artificial bearing area, which is able to support the progressive movement of the facet joints, was demonstrated. A lock-move (DE102016009670.5) which results from this consideration is able to be implanted into the interspinous space using cortical bone trajectories (Figure 5C). A precursor device was implanted in a 38-year-old male patient with a follow up time of four years, who had been operated 10 times on a prolapse in L5/S1, thereby damaging the facet joints. He refused stabilization. He was supplied with a monoblock prosthesis (Figure 5A) and a facet joint support based on polyaxial screw heads, which allowed a restricted translation (Figure 5B).
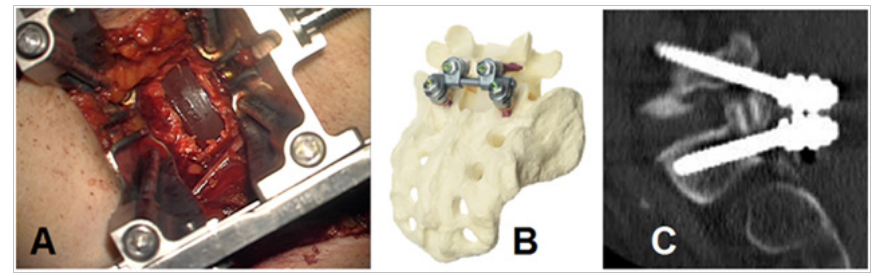

Figure 5 Functional replacement. A: implantation of the lumbar prosthesis B: principle of a primitive lock-move based on polyaxial screwheads $\mathrm{C}$ : postoperative CT-scan showing the vectors of the screws.

\section{Conclusion}

It is feasible to measure and conclusively simulate segmental movement patterns in order to provide mathematical-algorithmic theorems for the development of an interspinous device that restores optimal segmental height, movement characteristics and further supports the facet joints. In contrast, pedicle screw based dynamic support (topping-offs), does not allow dynamic adjustments of segmental height and therefore progressively can't emulate natural segmental movement according to the results of the above outlined surrogate model.

\section{Acknowledgments}

None.

\section{Conflicts of interest}

None. 